\title{
CORRECCIÓN DE RECTOCELE CON IVS POSTERIOR Y MALA TUNELZADA POR EL TABIQUE RECTOVAGINAL
}

\author{
Erasmo Bravo O. ${ }^{1}$, Bernardita Alamos L. ${ }^{a}$, Harald Riesle P. ${ }^{1}$, Aníbal Scarella Ch. ${ }^{1}$ \\ ${ }^{1}$ Unidad de Ginecología, Hospital Carlos Van Buren, Cátedra de Obstetricia y Ginecología - Universidad de Valparaíso.
}

a Interna de Medicina, Universidad de Valparaíso.

\section{RESUMEN}

Presentamos 50 pacientes portadoras de prolapso genital posterior sometidas a reparación con malla de prolene tunelizada y aplicación de cinta de prolene transelevador como profilaxis del prolapso de pared vaginal posterior, ingresadas al servicio de Ginecología del Hospital Carlos Van Buren entre Diciembre de 2004 y Febrero de 2006. El diagnóstico preoperatorio fue prolapso genital completo en 38 mujeres, prolapso de pared vaginal anterior y posterior en 8, prolapso de cúpula en 3 y prolapso de pared vaginal posterior en una. En 30 pacientes se asoció además incontinencia urinaria de esfuerzo. $92 \%$ de las pacientes evolucionan en forma satisfactoria en el seguimiento intra y postoperatorio con restauración de la anatomía del piso pelviano y sin complicaciones mayores. Creemos que es necesario un seguimiento mayor para validar en forma definitiva esta técnica, aún cuando en nuestro estudio este procedimiento resulta superior al que utiliza el "puente mucoso" para reparar la pared posterior.

\section{PALABRAS CLAVES: Rectocele, prolapso, malla tunelizada, tabique rectovaginal}

\section{SUMMARY}

We present 50 patients carrying later genital prolapse who went under repair with a prolene mesh and a posterior intravaginal sling as a prophylaxis of the posterior vaginal prolapsed wall, entered to the service of Gynecology of the Carlos Van Buren Hospital between December 2004 and February 2006. The preoperating diagnosis was complete genital prolapse in 38 women, prolapse of previous and later vaginal wall in 8 , prolapse of the cupula in 3 and prolapse of the later vaginal wall in one. In 30 patients urinary incontinencia to effort was associated in addition. $92 \%$ of the patients evolve in satisfactory form in the intra and postoperative pursuit with restoration of the anatomy of the pelvian floor and without greater complications. We believe that a greater pursuit is necessary to validate this technique completely, even though in our present study this procedure is superior to which uses the "mucous bridge" to repair the later wall.

\section{KEY WORDS: Rectocele, prolapse, tunnelized mesh, rectovaginal wall}

\section{INTRODUCCIÓN}

El prolapso genital es una entidad patológica frecuente dentro de la ginecología y su tratamiento ha ido evolucionando con técnicas menos invasivas y que presentan un menor número de complicaciones $(1,2)$.

Podemos definir al prolapso genital como una relajación generalizada del piso pelviano que puede manifestarse ya sea a través de la pared 
vaginal anterior como posterior, así como también asociarse $o$ no a incontinencia urinaria de esfuerzo.

En la generación de esta patología participan un gran número de factores etiológicos que en muchos casos dificultan el diagnóstico y la elección del tratamiento más adecuado para su corrección: entre éstos podemos mencionar factores mecánicos, neurológicos y trastornos del tejido conectivo. Asimismo, también podemos reconocer factores de riesgo como la edad, paridad, obesidad, constipación, cirugía pélvica previa, tabaquismo, enfermedades pulmonares crónicas y factores raciales que podrían estar involucrados en el desarrollo de éste. Dentro de estos últimos, es evidente que el embarazo y el parto implican un traumatismo directo al tabique recto-vaginal, a la musculatura y a las estructuras nerviosas y vasculares del piso pelviano $(3,4)$.

Con respecto al prolapso de pared vaginal posterior (PPVP), encontramos dos tipos de entidades que lo representan. Uno es el rectocele, que se define como la protrusión del recto a través de la pared vaginal posterior y el otro; el enterocele, el cual es el descenso de peritoneo e intestino a través del fondo de saco vaginal posterior hacia la vagina. Ambas patologías no constituyen una causa de mortalidad significativa, pero dada su alta frecuencia entre mujeres añosas y su variada sintomatología, sí representan una morbilidad trascendente en cuanto a la alteración en la calidad de vida de estas pacientes ya que entre los síntomas que producen se encuentran el estreñimiento, sensación de abombamiento vaginal, incontinencia de gases y fecal, vaciamiento rectal incompleto, dolor rectal y disfunción sexual (7-10).

Clásicamente, el PPVP se corregía quirúrgicamente con colporrafia posterior con plicatura de la fascia rectovaginal y miorrafia del músculo elevador del ano (11-17) pero se ha desistido en su realización debido a la alta tasa de recurrencia que presentó esta técnica.

Actualmente, el procedimiento quirúrgico planteado es el refuerzo de la pared vaginal posterior (PVP) colocando una malla de prolene que fortalezca la fascia recto-vaginal dañada y de esta manera restituya la tensión necesaria para mantener en su posición las estructuras aledañas. (5,1721). En un comienzo, esta malla era colocada con la técnica denominada de "puente mucoso" ante lo que se debía escindir la mucosa de la PVP. Hoy en día, la técnica se ha modificado en el Servicio de Ginecología del Hospital Carlos Van Buren de Valparaíso, debido a la complicación más frecuen- temente observada con el procedimiento anterior -como lo es la exposición de la malla y su posterior retiro- $y$ en vez se ha ideado tunelizarla entre vagina y recto sin abrir la mucosa.

Concomitantemente, y tal como lo describe Petros (6), se realiza además un IVS posterior (Posterior Intravaginal Slingplasty) o "Infracoccigeal Sacropexy", para reparar el prolapso de cúpula generalmente asociado (22). Esta técnica considera los conceptos definidos por De Lancey quien delimita tres niveles de sustentación útero-vaginal y a los cuales Petros apunta reparar evitando la procidencia de útero o el prolapso de cúpula vaginal.

Descrito lo anterior, el objetivo del presente trabajo es combinar la técnica del IVS posterior con la tunelización de una malla de prolene a través del tabique rectovaginal como tratamiento del PPVP, plasmar resultados a corto y mediano plazo en cuanto a complicaciones del procedimiento (intra y post-operatorias), y especialmente para determinar la superioridad en cuanto a menor riesgo de éstas y de recidiva frente a una técnica con malla instalada a través de lo descrito como "puente mucoso".

\section{MATERIAL Y MÉTODO}

Se reclutaron los datos de todas las pacientes con diagnóstico de prolapso de pared vaginal posterior operadas entre Diciembre de 2004 y Febrero de 2006 en forma retrospectiva y protocolizada. 50 mujeres con diagnóstico de PPVP fueron operadas en el Servicio de Ginecología del Hospital Carlos Van Buren durante ese período y con la técnica que se describirá posteriormente.

Las pacientes fueron evaluadas preoperatoriamente de acuerdo a la clasificación de BadenWalker (23), en posición de litotomía y con el máximo esfuerzo con maniobra de Valsalva.

En las pacientes con patología benigna de útero se realizó primero la histerectomía vaginal según técnica estándar (24) y además en aquellas, con el diagnóstico de prolapso de pared vaginal anterior con o sin incontinencia urinaria de esfuerzo (IUE) concomitante, se realizó la plastía anterior junto con la técnica de TOT (25) como corrección de la IUE cuando ésta estaba presente.

Técnica quirúrgica: Previo al procedimiento se administra un gramo de cefazolina endovenosa como profilaxis antibiótica. Se utiliza anestesia regional de tipo espinal o combinada epiduralespinal. Se ubica a la paciente en la mesa quirúrgica en posición de litotomía, utilizando pierneras 
con apoyo sólo en los pies, con lo cual se logra una excelente exposición perineal necesaria para la intervención, además de evitar la compresión vascular de extremidades inferiores. La técnica consiste en fijar una cinta de prolene a la cúpula vaginal en los puntos estimados de inserción de los ligamentos útero-sacros, cinta cuyos extremos se extraen bilateralmente por el espacio isquiorectal en dirección a un punto ubicado a $3 \mathrm{~cm}$ por fuera y atrás del ano, en donde se abandonan libre de tensión. El procedimiento comienza con la exposición transversal de la fascia de la pared vaginal posterior a 1,5 ó $2 \mathrm{~cm}$ por debajo de la cicatriz de la histerectomía anterior o borde de la colpotomía, disecando en forma lateral. Posteriormente se realiza una incisión puntiforme en la piel a $3 \mathrm{~cm}$ por fuera y atrás del ano en forma bilateral. Por ésta se introduce una aguja por el espacio isquio-rectal, traspasando el plano del elevador del ano y se exterioriza a través de la fascia rectovaginal en la zona expuesta previamente en la cúpula vaginal a nivel de las espinas ciáticas; un extremo de la cinta de prolene se ancla al ojal de la aguja y esta última se tracciona de regreso hacia el periné hasta exteriorizar el extremo de la aguja con la cinta. Se repite el procedimiento al lado contrario. Se cortan los extremos de la cinta y se abandona libre de tensión procurando que los extremos no lleguen al celular subcutáneo.

En la zona de la cúpula la onda de la cinta se fija con puntos separados de Vicryl 1 a la pared vaginal en las zonas estimadas de inserción de los ligamentos útero-sacros. Finalmente, con el fin de reforzar la fascia recto-vaginal de la pared posterior de la vagina se utiliza una malla de prolene la que se coloca entre vagina y recto, tunelizándola a través del espacio entre ambos sin abrir mucosa vaginal. El instrumento utilizado para la colocación de la cinta consiste en una aguja curva, de acero inoxidable, reutilizable y de punta roma.

\section{RESULTADOS}

Se operó un total de 50 pacientes, cuyos rangos de edad entre 36 y 87 años. Las características de edad y paridad se describen en las Tablas I y II.

El diagnóstico preoperatorio de las pacientes se expone en la Tabla III en donde observamos que el prolapso genital completo estaba presente en 38 mujeres, prolapso de pared vaginal anterior y posterior en 8 , prolapso de cúpula en 3 y prolapso de pared vaginal posterior en una. En 30 pacientes se asoció además IUE.
Tabla I

\section{EDAD DE LAS PACIENTES}

\begin{tabular}{lr}
\hline Rangos en años & $n$ \\
\hline$<40$ & 2 \\
$40-49$ & 9 \\
$50-59$ & 14 \\
$60-69$ & 12 \\
$70-79$ & 7 \\
$80-89$ & 6 \\
$>89$ & 0 \\
\hline Total & 50 \\
\hline
\end{tabular}

Edad promedio

59,5

Tabla II

PARIDAD DE LAS PACIENTES

\begin{tabular}{lr}
\hline Paridad & $n$ \\
\hline Nuligesta & 3 \\
M1 - M3 & 26 \\
M4 o más & 21 \\
\hline Total & 50 \\
\hline
\end{tabular}

M: multípara.

Tabla III

DIAGNÓSTICOS PRE- OPERATORIOS

\begin{tabular}{lr}
\hline Diagnósticos & $n$ \\
\hline Prolapso genital completo & 38 \\
Prolapso pared vaginal anterior y posterior & 8 \\
Prolapso pared vaginal posterior & 1 \\
Prolapso cúpula vaginal & 3 \\
Incontinencia urinaria esfuerzo o mixta & 30 \\
\hline Total de pacientes con prolapso & 50 \\
\hline
\end{tabular}

Las cirugías realizadas se describen en la Tabla IV. Se observa que en 3 pacientes se realizó sólo corrección de rectocele y a 4 además se les asoció histerectomía vaginal. En 41 pacientes tuvo también que corregirse el prolapso anterior con la plastía correspondiente y en 2 mujeres se realizó plastía anterior y corrección de rectocele.

El tiempo operatorio de las distintas cirugías fue muy variable, con un valor promedio de 1 hora y 46 minutos (rango: 1 hora - 2 horas y 50 minutos). El tipo de anestesia utilizada fue 35 pacientes con anestesia espinal y en las 15 restantes anestesia combinada epidural-espinal. El tiempo 
Tabla IV

CIRUGÍA REALIZADA

\begin{tabular}{lr}
\hline Cirugía realizada & $n$ \\
\hline Corrección sólo de rectocele & 3 \\
CR + histerectomía vaginal & 4 \\
CR + histerectomía vaginal + plastía anterior & 41 \\
Corrección rectocele + plastía anterior & 2 \\
\hline
\end{tabular}

Total cirugías 50

CR: Corrección de rectocele.

de hospitalización promedio fue 2,5 días (rango: 2 - 5 días).

Hubo una complicación intraoperatoria que correspondió a lesión del detrusor, y 3 postoperatorias ( 1 recidiva de rectocele, 1 dehiscencia de la zona central de la tunelización posterior y 1 paciente que manifestó dolor espontáneo y a la palpación de IVS posterior). Con estas 4 complicaciones obtenemos un $8 \%$ del total.

\section{DISCUSIÓN}

Esta es la primera comunicación en nuestro medio de una experiencia con la técnica conjunta entre IVS posterior y malla tunelizada a través del tabique rectovaginal para corrección de prolapso de pared vaginal posterior.

A pesar de ser una recolección de datos no lo suficientemente extensa como para afirmar con certeza estadística la eficacia de este método, sí podemos plantear al menos con esta casuística que la metodología demuestra una menor morbilidad y molestias postoperatorias, no adiciona instrumental extra, resulta en similar tiempo operatorio y requiere de un ginecólogo entrenado en cirugía urogenital.

Es importante destacar que esta técnica se basa en consideraciones anatómicas que reparan los defectos que permiten el prolapso de una manera menos invasiva con respecto a la del "puente mucoso" al sólo tunelizar la malla en vez de escindir la mucosa, lo cual obviamente disminuye el número de exposiciones de este elemento debido a que aminora la reacción tisular habitual desarrollada frente a un cuerpo extraño ya que no se realiza corte ni sutura sobre la pared vaginal posterior.

Las pacientes fueron en su gran mayoría multíparas y, en general, con antecedentes de partos vaginales. Podemos decir también que al momento de la cirugía bordeaban la peri y post- menopausia, dato que no difiere de lo descrito como factor de riesgo en la pacientes con prolapso $(20,26,27)$.

En relación a los diagnósticos pre-operatorios, el prolapso genital completo estuvo presente en el $76 \%$, el resto correspondió a prolapsos que comprometían ya sea las paredes vaginales (en conjunto o por sí solas) o la cúpula solamente. Además, como era de esperarse, la IUE representó una patología importante relacionada a esta relajación del piso pelviano, estando presente en el $60 \%$ de ellas (27).

La anestesia regional utilizada en todas nuestras pacientes no presentó complicaciones, lo que reafirma lo seguro de este procedimiento para este tipo de cirugías.

El tiempo operatorio promedio de 1 hora 46 minutos nos parece adecuado para la fase en la que se encuentra la realización de esta técnica, dado que es una práctica nueva en etapa inicial de aprendizaje.

El tiempo de estadía hospitalaria fue de 2,5 días, nos parece que a pesar de tener cirugías asociadas éste es breve, y que esta técnica no suma más días a la cirugía ginecológica concomitante.

Las complicaciones intra y post-operatorias fueron poco frecuentes y leves. Es importante destacar el bajo número de casos en que se observó la exposición de la malla en la cúpula vaginal, lográndose el objetivo planteado al idear esta técnica, y con ello no sólo aminorar la morbilidad asociada a la cirugía sino que también la recidiva del prolapso y la necesidad de una reintervención.

Por todo esto, en términos de complicaciones, tiempo operatorio y días de hospitalización la reparación quirúrgica del prolapso de pared vaginal posterior con cinta transelevador y malla de prolene tunelizada, en este estudio, es una técnica segura, fácil de realizar y superior en cuanto a resultados a la que utiliza el "puente mucoso" asociado a IVS posterior (28). Aún cuando creemos que es necesario un seguimiento mayor para validar en forma definitiva la técnica.

\section{BIBLIOGRAFÍA}

1. Braun H, Rojas I, González F, Fernández M. Prolapso genital severo: Consideraciones clínicas, fisiopatológicas y de técnica quirúrgica al momento de su corrección. Rev Chil Obstet Ginecol 2004; 69(2): 149-56.

2. Olsen AL, Smith VJ, Bergstrom JO, Colling JC, Clark AL. Epidemiology of surgically managed pelvic organ 
prolapse and urinary incontinence. Obstet Gynecol 1997;89:501-6.

3. Mellegren A, Anzen B, Nilsson BY, et al. Results of rectocele repair - a prospective study. Dis Colon Rectum 1995;38:7-13.

4. Infantino A, Masin A, Melega E, Dodi G, Lise M. Does surgery resolve outlet obstruction? Int J Colorectal Dis 1995;10:97-100.

5. Abramos Y, Gandhi S, Goldberg R, Botros S, et al. Site-specific rectocele repair compared with standard posterior colporrhapy. Obstet Gynecol 2005; 105(2):314-8.

6. Silva A, Ferrada L, Arroyo C, Nalbandian K. IVS posterior (posterior intravaginal slingplasty) para el tratamiento de prolapso de cúpula vaginal o procidencia del útero: serie de casos. Rev CHIL OBStET GINECOL 2004;69(6):419-23.

7. Lubowski DZ, King DW. Obstructed defecation: Current status of pathophysiology and management. Aust NZ J Surg 1995;65:87-92.

8. Kahn MA, Stanton SL. Techniques of rectocele repair and their effects on bowel function. Int Urogynecol J 1998;9:37-47.

9. Glavind $\mathrm{K}$, Madsen $\mathrm{H}$. A prospective study of the discrete fascial defect rectocele repair. Acta Obstet Gynecol Scand 2000;79:145-7.

10. Jackson SL, Weber AM, Hull TL, Mitchinson AR, Walters MD. Fecal incontinence in women with urinary incontinence and pelvic organ prolapse. Obstet Gynecol 1997;89:423-7.

11. Cundiff GW, Weidner AC, Visco AG, Addison WA, Bump RC. An anatomic and functional assessment of the discrete defect rectocele repair. Am J Obstet Gynecol 1998;179:1451-7.

12. Glavind $\mathrm{K}$, Madsen $\mathrm{H}$. A prospective study of the discrete fascial defect rectocele repair. Acta Obstet Gynecol Scand 2000;79:145-7.

13. Kahn MA, Stanton SL. Posterior colporrhaphy: Its effects on bowel and sexual function. BJOG 1997; 104:82-6.

14. Kenton K, Shott S, Brubaker L. Outcome after rectovaginal fascia reattachment for rectocele repair. Am J Obstet Gynecol 1999;191:1360-4.

15. Lopez A, Anzen B, Bremmer S, et al. Durability of success after rectocele repair. Int Urogynecol J 2001;12:97-103.
16. Mellegren A, Anzen B, Nilsson BY, et al. Results of rectocele repair - a prospective study. Dis Colon Rectum 1995;38:7-13.

17. Miklos JR, Kohli N, Moore R. Levatorplasty release and reconstruction of rectovaginal septum using allogenic dermal graft. Int Urogynecol J Pelvic Floor Dysfunct 2002;13:44-6.

18. Watson SJ, Loder PB, Halligan S, Bartram Cl, Kamm MA, Phillips RK. Transperineal repair of symptomatic rectocele with Marlex mesh: A clinical, physiological and radiologic assessment of treatment. J Am Coll Surg 1996;183:257-61.

19. Sand PK, Koduri A, Lobel RW, Winkler HA, Tomezsko J, Culligan PJ, Goldberg R. Prospective randomised trial of polyglactin 910 mesh to prevent recurrence of cystocele and rectoceles. Am J Obstet Gynecol 2001;184:1357-64.

20. Goh JTW, Dwyer PL. Effectiveness and safety of polypropylene mesh in vaginal prolapse surgery. Int Urogynecol J 2001;12:S90.

21. Planells Roig M. Prospective analysis of marlex mesh repair for symptomatic rectocele with obstructive defecation. Rev Esp Enferm Dig 2002; 94(2): 67-77.

22. Atlanta Center for Laparoscopic Urogynecology. John R Miklos, Robert D. 2000 -2003. Hallado en : www.tvtsling.com/lap_proc8a_Cont. php .

23. Baden WF, Walker TA, Lindsey JH. The vaginal profile. Tex Med 1968;64:56-9.

24. Bravo E, Frank C, Prado J, Carmona A, et al. Histerectomía vaginal en ausencia de prolapso. Rev Chil Obstet Ginec 1998;63(4):257-62.

25. Bravo E, Benett C, Parada R, et al: Histerectomía vaginal y corrección de incontinencia urinaria de esfuerzo con técnica de TVT Obturador. Rev Chil Obstet Ginec 2003;68(4):283-86.

26. Peters WA III, Smith MR, Drescher CW. Rectal prolapse in women with other defects of pelvic floor support Am J Obstet Gynecology 2001;184(7):148894.

27. Partoll LM. Efficacy of tension-free vaginal tape with other pelvic reconstructive surgery. Am J Obstet Gynecol 2002;186(6):1295-8.

28. Goh JT, Tjandra JJ, Carey MP. How could management of rectoceles be optimized? ANZ J Surg 2002;72(12):896-901. 